\title{
Fan-shaped Spectrums of Species and Paleopolyploidy for Crossbreeding Evolution
}

\author{
Li-Yuan Liu Dong-Sheng Guo \\ College of Life Science, Beijing Normal University, Beijing, China, 100875
}

\begin{abstract}
Although Darwin's evolutionary mutation theory has been widely accepted, many endeavors tried to challenge it. With more and more observation of successful hybridization and hybrids, the sexual isolation between species has become vague. The mechanism of evolution has been expanded from the classical model of evolution to multiple routes of speciation. Furthermore, a fundamental crossbreeding theory has been raised and proved by two lines of evidences: paleopolyploidy and fan-shaped spectrum of species. Ancient genome duplications are widespread throughout eukaryotic lineages, particularly in plants. The genome polyploidization can break through the sexual incompatibility between diploid counterparts to hybridize and produce new species. By comparing characteristics, all species in every taxon, both in the extinct fossil and extant organisms, can be arranged into fan-shaped spectrum according to their similarity: left primitive type-middle advanced type-right primitive type. The species are primitive at the two ends and advanced at the middle. The primitive two species always resemble two types of more primitive species that can be confirmed as their ancestors respectively, and the middle species is half similar to the two ancestors respectively. These suggest that the species in the spectrum come from two different ancestors by crossbreeding and gene combination. As a sum, advanced species originated from crossbreeding of two primitive ancestors, by major method of polyploidization, and proved by results of fan-shaped spectrum of species. Then, sex is the cause, force and opportunity for evolution.
\end{abstract}

Key Words: Evolution, Speciation, Crossbreeding, Hybridization, Species spectrum, Polyploidization, Mutation 
The evolutionary theory of Darwin, both on mutation and species, has been challenged by many new evidences. It is almost cannot imaged that a single-celled organism could become a human body through random mutation. It is also cannot believed that we succeeded from making mistakes (mutation) as in Darwinism, in which the more errors, the more success, and the numerous errors, the great success.

Many researchers tried to explore the mechanism of evolution outside of the Darwinism, even raising a new crossbreeding theory to replace Darwin' mutation theory. The new theory is strongly supported by two lines of evidences: fan-shaped spectrum of species and paleopolyploidy. Then the mutation is not so important again as ever thought, but the crossbreeding is far more important than ever accepted.

A hybrid, or crossbreed, is the result of combining the qualities of two organisms of different breeds, varieties, species or genera through sexual reproduction. Here, "crossbreed" (verb) is used for more widespread crossing phenomena across more different breeds or species. But "hybridize" belong to the known crossing phenomena.

\section{Sexual barrier between species}

Since the evolutionary synthesis, a dominant definition of species in the evolutionary biology has been the so-called "biological species concept". Under this concept, members of the same species "actually or potentially interbreed" (Mayr 1963), whereas members of different species cannot do so (e.g. crossbreed). Thus there is a clash between two views of species: "one is based on the pattern of gene flow, and the other on the maintenance of a cluster of phenotypes " (Barton and Hewitt 1989).

\section{Mechanisms of reproductive isolation}

A variety of mechanisms limit the success of hybridization, including the large genetic difference between most species. Animal species are reproductively isolated by strong barriers to hybridization, which include morphological differences, differing times of fertility, mating behaviors and cues, and physiological rejection of sperm cells or the developing embryo. 
Animal interspecific hybrids are bred normally from within the same genus. The offspring display traits and characteristics of both parents, but are often sterile, preventing gene flow between the species (Keeton 1980). Sterility is often attributed to the different number of chromosomes between the two species. A typical example is mules or hinnies with 63 chromosomes. However, fertility in about 4 female mules has been reported (e.g.: Rong 1988).

In plants, some barriers to hybridization include blooming period differences, different pollinator vectors, inhibition of pollen tube growth, somatoplastic sterility, cytoplasmic-genic male sterility and structural differences of the chromosomes.

\section{Hybridization}

In the past, hybridization was viewed as a secondary phenomenon of little or no evolutionary importance. Alternatively, hybrids and hybridization can be viewed as natural intermediate stages of a gradual process of differentiation, possibly in sympatry or parapatry (Arnold and Mallet 1938; Barton and Hewitt 1989). Many studies have been done on hybrid zones (Barton and Hewitt 1989; Harrison 1993; Butlin 1998), but the hybridization is considered having little relevance to interspecific hybridization and the hybrids may be sterile or inviable to produce any offspring.

Introgressive hybridization have been described and defined in plants in 1938 (Anderson and Hubricht 1938). In recent 20 years, some researchers have been made in understanding the species boundary in animals both below and above the level of species. Below the species level, forms are known which remain distinct in spite of potential or actual gene flow. Above the species level, they are beginning to appreciate that hybridization, while rare on a per-individual basis, is a regular and probably important occurrence in nature (Arnold 1997; Grant 1992; Allendorf et al. 2001; Seehausen 2003) On average, at least $10 \%$ of animal species and maybe $25 \%$ of plant species are known to hybridize in nature, although the fraction of species that hybridize may be much higher in rapidly radiating groups (Mallet 2005).

Mallet et al reported that the interspecific hybrids occur regularly in two 
genus of butterflies (Heliconius and eueides in Lepidoptera: Nymphalidae) in the wild: $26-29 \%$ of the species of heliconiina are involved (Mallet et al. 2007). On Daphne Major Island, the Darwin's Finches have taken place complex intercrossing between several species and backcrossing. The final Introgressive hybridization has led to the formation of a new lineage after an extinctive death in the drought of 2003-2005 (Grant and Grant 2014). In the great cats of the genus panthera (Henrique et al. 2017), Henrique et al observed pervasive genealogical discordance, caused by both incomplete lineage sorting and complex patterns of historical interspecific hybridization. The intercrossing taken placed after the lineages have been separated. These indicate that sexual and dioecious animals also obey the same fundamental laws of gene flow and introgression as plants and bacteria.

\section{Mutation for speciation}

It is widely believed that mutations arise continuously and without any consideration for their utility. But more and more controversial papers have been published. Some experiments suggested that cells may have mechanisms for choosing which mutations will occur. Cairns found that when they placed bacteria that could not digest the milk sugar lactose in an environment where that sugar was the sole food source, the cells soon evolved the ability to convert the lactose into energy (Cairns et al. 1988).

Witkin reported that accidentally irradiated millions of $E$. coli with a lethal dose of ultraviolet light. They were all dead except for one, in which four bacterial cells had survived and continued to grow (Witkin 1946). Somehow, those cells were resistant to UV radiation. These are now known as the SOS response. The harsh environments cause nonrandom mutations and help bacteria evolve advantageous traits more quickly in stressful environments. It's a totally new way that the environment can have an impact on the genome to allow adaptation in response to need (Hull et al. 2017; Frenk et al. 2017). These indicate that the genome itself may have any kind of intelligence to keep "alive" and inheritance. 


\section{Back to Mendel and crossbreeding}

Under the shadow of Darwin's success is Mendel's illuminating work (Darwin 1872; Mendel 1865). Mendel's work, however, should be only partially rediscovered in the 19th century. It was regarded as a supplement to Darwin's theory: the variation of characteristics is based on gene mutation. Actually, Mendel's experiments undoubtedly revealed that new characteristics in new species/subspecies came from combination of parent's genes by interbreeding between two different species/subspecies. Then, an expanded argument for speciation is not from gene mutation in Darwinism, but from gene combination by crossbreeding or Hybridization.

\section{Paleopolyploidy and Crossbreeding}

\section{Problem of chromosome numbers}

Mutation only can change a few number of codes in the genes within the chromosome, but never can produce a new different chromosome. Thus, by mutation, the numbers of chromosomes do not increase any more. Apparently, this is not coincident with the organisms.

The numbers of chromosomes in diffident species are different and vary largely. Even in same family or same genus, there are quite distinct. For instance, in the same family of canidae, dog has 78 chromosomes, bengal fox has 60 chromosomes, but American marten has 38 chromosomes. In the rodents, rat has 42 chromosomes, but crab-eating rat has 92 chromosomes that are highest for a mammal. Atlas blue, a butterfly, has the highest number of chromosomes (448-452) in the non-polyploid eukaryotic organisms.

Undoubtedly, animals are far more complex than plants, and vertebrates are far more advanced than invertebrates. But we almost cannot differentiate them from the numbers of chromosomes. The most advanced and most complex human beings only have 46 chromosomes, but the primitive vertebrate, carps, have 104 chromosomes. The organisms with more chromosomes are not in the complex animals, but in the simpler plants. For instance, rattlesnake fern has 184 chromosomes and ophioglossum have 1200 or 1260 chromosomes. The latter is the highest known chromosome numbers. 
To explain such irregular differences in the number of chromosomes, the best answer is perhaps that the species come from crossbreeding from different ancestors with different numbers of chromosomes and result in different combination and distribution of chromosomes. So the number of chromosomes can increase by co-existence of chromosomes after every fertilization, or decrease by lost some chromosomes. This is particularly true in cervidae.

Cervidae have considerable diversity of karyotypes, despite their morphological similarity (Wang and Lan 2000). The Indian muntjac possesses the lowest diploid chromosomal number in mammals $(2 n=6$ for females $[F]$ and 7 for males [M]), whereas the Chinese muntjac has a $2 n$ number of 46 in both sexes. These two species, however, can produce viable F1 hybrids ( $2 n=$ 27) in captivity, and partial spermatogenesis was observed in hybrids. Other karyotyped species have intermediate numbers of chromosomes; for example, $2 n=8 \mathrm{~F}, 9 \mathrm{M}$ in muntiacus crinifrons, and $2 \mathrm{n}=13 \mathrm{~F}, 14 \mathrm{M}$ in muntiacus feae. All these must be results of hybrids and the chromosome numbers have been changed all the time.

\section{Polyploidy and paleopolyploidy}

Plant species often hybridize and the resulting hybrids are fertile more often. Many plant species are the result of hybridization, combined with polyploidy, which duplicates the chromosomes. Chromosome duplication allows orderly meiosis and so viable seed can be produced (Goulet et al 2016).

Paleopolyploidy is the result of genome duplications which occurred at least several million years ago. Such an event could either double the genome of a single species (autopolyploidy) or combine those of two species (allopolyploidy). Because of functional redundancy, genes are rapidly silenced or lost from the duplicated genomes. Most paleopolyploids, through evolutionary time, have lost their polyploid status through a process called diploidization, and are currently considered diploids.

Ancient genome duplications are widespread throughout eukaryotic lineages, particularly in plants. It has been found that almost all flowering plants have undergone at least one round of genome duplication at some 
point during their evolutionary history (Bowers et al. 2003; Keith Adams 2013). Compared with plants, known paleopolyploidy is less in the animal kingdom. It has been identified mainly in amphibians and bony fishes. The idea that vertebrates share a common whole genome duplication is known as the " $2 R$ Hypothesis".

\section{2R Hypothesis}

The hypothesis of vertebrate paleopolyploidy was proposed by Susumu Ohno as early as the 1970s. He reasoned that the vertebrate genome could not achieve its complexity without large scale whole-genome duplications. The "two rounds of genome duplication" hypothesis (2R hypothesis) came about, which is a hypothesis that the genomes of the early vertebrate lineage underwent two complete genome duplications, and thus modern vertebrate genomes reflect paleopolyploidy (Hokamp et al. 2003).

The $2 \mathrm{R}$ hypothesis saw a resurgence of interest in the 1990s for two reasons. First, gene mapping data in humans and mice revealed extensive paralogy regions - sets of genes on one chromosome related to sets of genes on another chromosome in the same species, indicative of duplication events in evolution (Lundin 1993). Paralogy regions were generally in sets of four. Second, cloning of Hox genes in amphioxus revealed presence of a single Hox gene cluster, in contrast to the four clusters in humans and mice (Garcia-Fernández 1994). These two lines of evidence suggest that two genome duplications occurred in the ancestry of vertebrates. A new study generated the sea Lamprey genetic map, which yielded strong support for the hypothesis that a single whole-genome duplication occurred in the basal vertebrate lineage, preceded and followed by several evolutionarily independent segmental duplications that occurred over chordate evolution (Smith 2015).

\section{Advantage of allopolyploids}

The allopolyploids arise as a result of the hybridization of two related species, which are believed to be much more prevalent in nature, possibly because allopolyploids inherit different genomes, resulting in increased 
heterozygosity, and therefore higher fitness (Soltis 2000). These different genomes result in an increased likelihood of large genomic reorganizations, which can be either deleterious, or advantageous. The latter include:

1) Genome diversity Genome doubling provided the organism with redundant alleles that can evolve freely with little selection pressure. The duplicated genes can undergo neofunctionalization or subfunctionalization which could help the organism adapt to the new environment or survive different stress conditions.

2) Heterosis Polyploids often have larger cells and even larger organs. Many important crops, including wheat, maize and cotton, are paleopolyploids, which were selected for domestication by ancient people and are widely breeding today.

3) Speciation It has been suggested that many polyploidization events created new species, via a gain of adaptive traits, or by sexual incompatibility with their diploid counterparts.

\section{Fan-shaped Spectrum of Species and Crossbreeding}

In a new book, New Theory of Species Evolution, an evolutionary theory of crossbreeding is emphasized as a basic theory for evolution: advanced species originated from crossbreeding of two primitive species of different lineages (Liu 2016).

\section{Difficulties of the one-single ancestor}

According to Darwinism's mutation theory, all animals share a single common ancestor (Darwin 1872). But as describing in any zoological book, no one such ancestor can be confirmed exactly, because while some traits resemble to a presumed ancestor, some other traits are completely dissimilar. Such contradiction always existed between almost all presumed ancestors and descendants. In many cases, different authors presumed different ancestors for same species and the disagreement have been continued forever. For instance, the arthropods have been presumed to be descends from annelids, nematods, or mollusks respectively. The arguments indicate a phenomenon for 
a species, in which a part of its traits obviously come from one ancestor while other traits prominently come from another ancestor. Actually, it is a common phenomenon that when a species resembles one ancestor, it always possesses traits that are quite unlike that ancestor, but resembles another ancestor.

\section{Spectrums of species, involving two ancestors}

After analyzed almost all protists, all animal and plants from fossil forms and extant varieties and found that species always constitute a fan-shaped, successive series or spectrum at every level of taxon (e.g. Class, Order or Family) according to their similarity of characteristics. For instance, all nine species in a class of animals can be arranged into a spectrum as:

$$
A_{1}-B_{2}-C_{3}-D_{4}-E_{5}-F_{6}-G_{7}-H_{8}-I_{9}
$$

The adjacent species are most similar. Moreover, the species at the two ends of the spectrum (i.e. $A_{1}$ and $I_{9}$ ) highly resemble to two taxa of species in lower or more primitive class of (e.g. $P_{1}$ and $P_{2}$ ) that can be confirmed as their ancestors respectively. The species in the middle of the spectrum (i.e. $E_{5}$ ) half resembles to the two ancestors $\left(P_{1}\right.$ and $\left.P_{2}\right)$ respectively. These indicate that the species in the spectrum have two different ancestors of different lineages. The free combination of traits from both ancestors can produce the spectrum.

The proportion of traits from the two ancestors decide the descendant's position in the spectrum. The species with traits mainly from one ancestor is positioned at the left and the right end of the spectrum respectively (i.e. $\mathbf{A}_{\mathbf{1}}$ or I), while those with equal amounts of traits from both ancestors are positioned toward the middle of the spectrum (i. e. $E_{5}$ ). Others are arranged between the middle and left end of the spectrum (i.e. $B_{2}-D_{4}$ ) or between the middle and the right end of the spectrum (i.e. $\mathrm{F}_{6}-\mathrm{H}_{8}$ ). Such spectrum always can be found in any taxon.

Such spectrum can be easily understood in a family with many children, in which one may be very father-like and another may be very mother-like, others are partly father-like or mother-like. Someone may be not father-like and mother-like, actually, half-like respectively. 


\section{Crossbreeding model and Crossbreeding equation}

Apparently, more advanced species (new species) came from the combination of two primitive species (ancestors) from different lineages upon the spectrum. The way of combination is no other method than crossbreeding in the natural conditions. This process can be simply indicated as:

The discovery of fan-shaped spectrum for a taxon

$\rightarrow$ Two quite different primitive species in a spectrum or a taxon

$\rightarrow$ Two different ancestors from different lineages

$\rightarrow$ Crossbreeding

This mechanism can be simplified to express as mathematics-like equations in theoretical mode:

$P_{1}+P_{2} \rightarrow A_{1}, B_{2}, C_{3}, D_{4}, E_{5}, F_{6}, G_{7}, H_{8}$, I9 (Summed equation)

$\mathbf{P}_{1} \rightarrow \mathbf{A}_{\mathbf{1}}$ (Proved by characteristics with high similarity)

$\mathbf{P}_{2} \rightarrow \mathrm{I}_{\mathbf{9}} \quad$ (Proved by characteristics with high similarity)

$\mathbf{P}_{1} \approx A_{1} ; P_{2} \approx 19 ; \quad E_{5}=P_{1} / 2+P_{2} / 2 ;$

(Typical primitive type) (Typical middle type)

$B_{2} \approx P_{1} 7 / 8+P_{2} 1 / 8 ; \quad C_{3} \approx P_{1} 6 / 8+P_{2} 2 / 8 ; \quad D_{4} \approx P_{1} 5 / 8+P_{2} 3 / 8$

(Left primitive type) (Left intermediate type) (Low-middle type)

$\mathrm{H}_{8} \approx \mathrm{P}_{2} 7 / 8+\mathrm{P}_{1} 1 / 8 ; \quad \mathrm{G}_{7} \approx \mathrm{P}_{2} 6 / 8+\mathrm{P}_{1} 2 / 8 ; \quad \mathrm{F}_{6} \approx \mathrm{P}_{2} 5 / 8+\mathrm{P}_{1} 3 / 8$.

(Right primitive type) (Right intermediate type) (Right-middle type)

Furthermore, the free combination of traits from both ancestors or parents can be illustrated in figure 1, which are mosaic of characteristics from both parents or two ancestors. However, although free combination, the characteristics in a spectrum do not always change gradually. The mosaic traits, with so-called advanced traits in primitive species and primitive traits in advanced species, always exist together. The free combination is opportunity, but not results, because not all opportunities can occur and not all combinations can succeed. Note, the characteristics represent the phenotypes, but not the genomes. 


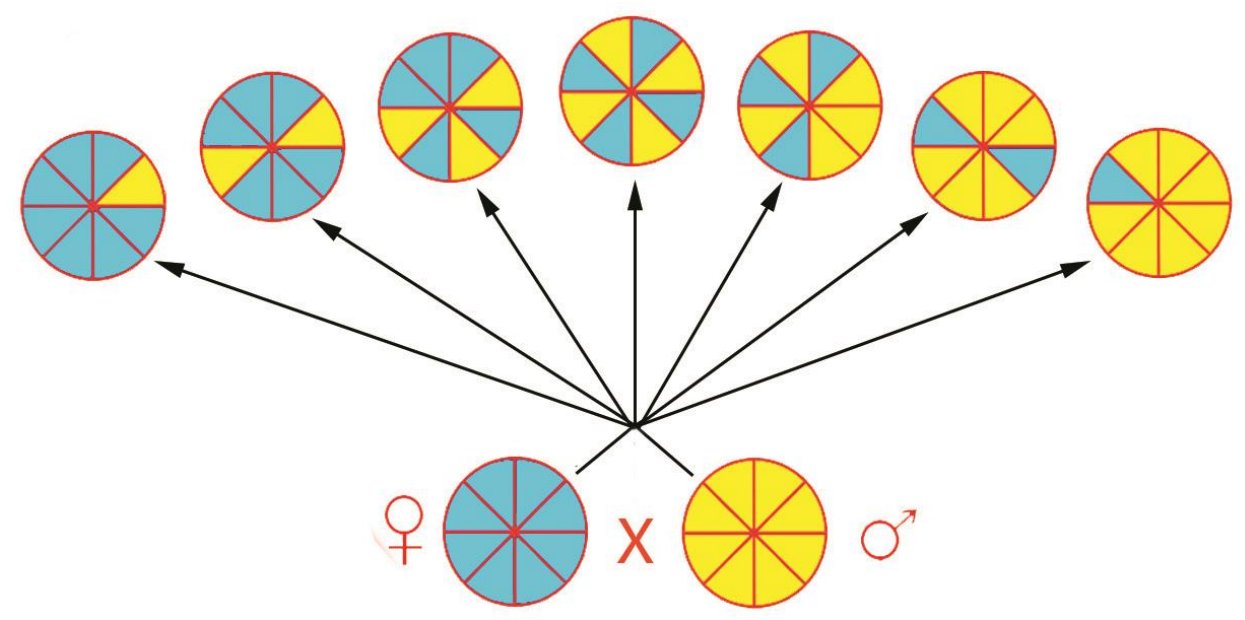

Fig. $1 \mathrm{~A}$ fan-shaped spectrum of species with possible opportunities or results from the free combination of characteristics after crossbreeding. Note, the mosaic-like distribution of characteristics.

\section{Normal Distribution of species}

In the probability theory of Statistics, Normal (or Gaussian) Distribution is a continuous probability distribution, with a few members at the high and low ends and many in the middle, as a bell curve. Many common attributes follow normal distribution. Here the descendants of crossbreeding, or the spectrum, from random combination also roughly follow the Normal Distribution, in which the middle type always have highest success rate or high race density, and the primitive type in two ends always have lower opportunity to survive and lower race numbers or density. Therefore, a spectrum of species shows a Normal Distribution (Fig. 2).

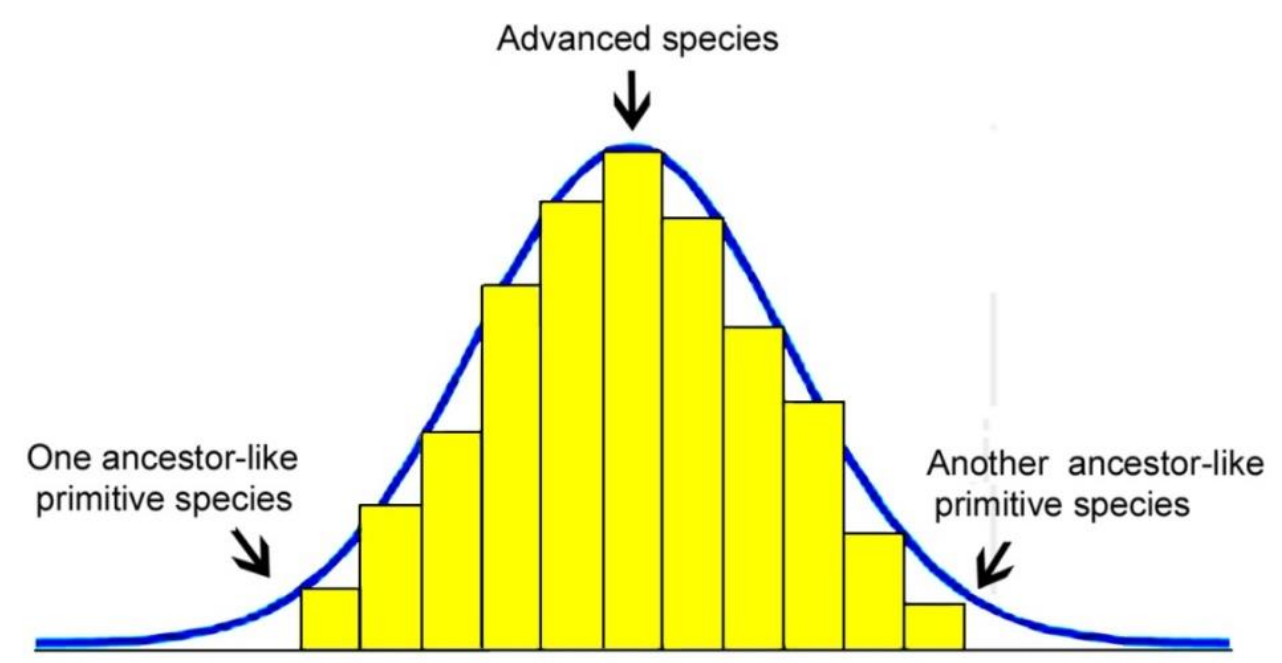

Fig. 2 Normal Distribution-like occurrences of new species after crossbreeding. 


\section{Evolutionary steps in animals}

All origins of protists and animals (exception of marsupials and the South American ungulates) are described and proved as above spectrum and equation in the book (Liu 2016). Next are some equations.

Origin of Protists:

1) Bacteria \Cyanobacteria + Archaea \Halobacteria $\rightarrow$ Algae

2) Archaea \Thermoplasma or Pyrodictium + Bacteria \Planctomycetes $\rightarrow$

Amoeboids, Zooflagellates and Metamonads

Origin of Invertebrates:

3) Ctenophores+ Entoprocts/Medusa $\rightarrow$ Echinoderms

4) Entoprocts + Turbellarians $\rightarrow$ Mollusks

5) Mollusks \Bivalves + Annelids $\backslash$ Polychaetes $\rightarrow$ Arthropods

\section{Origin of Chordates:}

6) Bivalves+ Nematoda $\rightarrow$ Urochordata (Tunicates), Cephalochordata (Amphixous etc.)

7) Thelodontids+Anaspids $\rightarrow$ Chondrichthyes: Sharks, Acanthodians, Chimaeras etc.

8) Antiarch + Osteolepis $\rightarrow$ Labyrinthodonts and Fish-formed Amphibians

9) Anthracosaurs +Temnospondyls $\rightarrow$ Anapsida

10) Ornithosuchians $\backslash$ Lagosuchus + Primitve Lizards $\rightarrow$ Saurischian Theropods, Birds etc.

11) Theriodonts $\backslash$ Cynognathus + Primitive Lizards $\rightarrow$ Most Primitive Mammals

12) Condylarths + Rodents $\rightarrow$ Ungulates

13) Lemurs + Bears $\rightarrow$ Anthropoids

The evolution of vertebrates notably occurred through several levels and steps. For instance, the evolution of amphibians had two crossbreeding stages. The evolution of reptiles had three crossbreeding stages: the most advanced reptiles, for instance, dinosaurs, birds and crocodilians, occurred in the third stage. But the evolution of mammals had four crossbreeding stages: the rodents, ungulates, and carnivores occurred in the third stage; the most advanced mammals, our anthropoids, occurred in the fourth stage, one more stage than the reptiles. 


\section{Crossbreeding examples in animals}

The species spectrum and crossbreeding theory can be illustrated by two examples. In the full spectrum of arthropods, ostracoda with its two halves of carapace resembles bivalves of mollusks and is placed at lowest end of the spectrum; onychophoran with segmented, worm-like body is quite similar to annelids (i.e. polychaetes) and placed at highest end of the spectrum; insects and crustaceans are arranged in the middle for their annelid-like abdomen and bivalve-like thorax. Thus, arthropods came from crossbreeding between bivalves and annelids (Fig. 3).

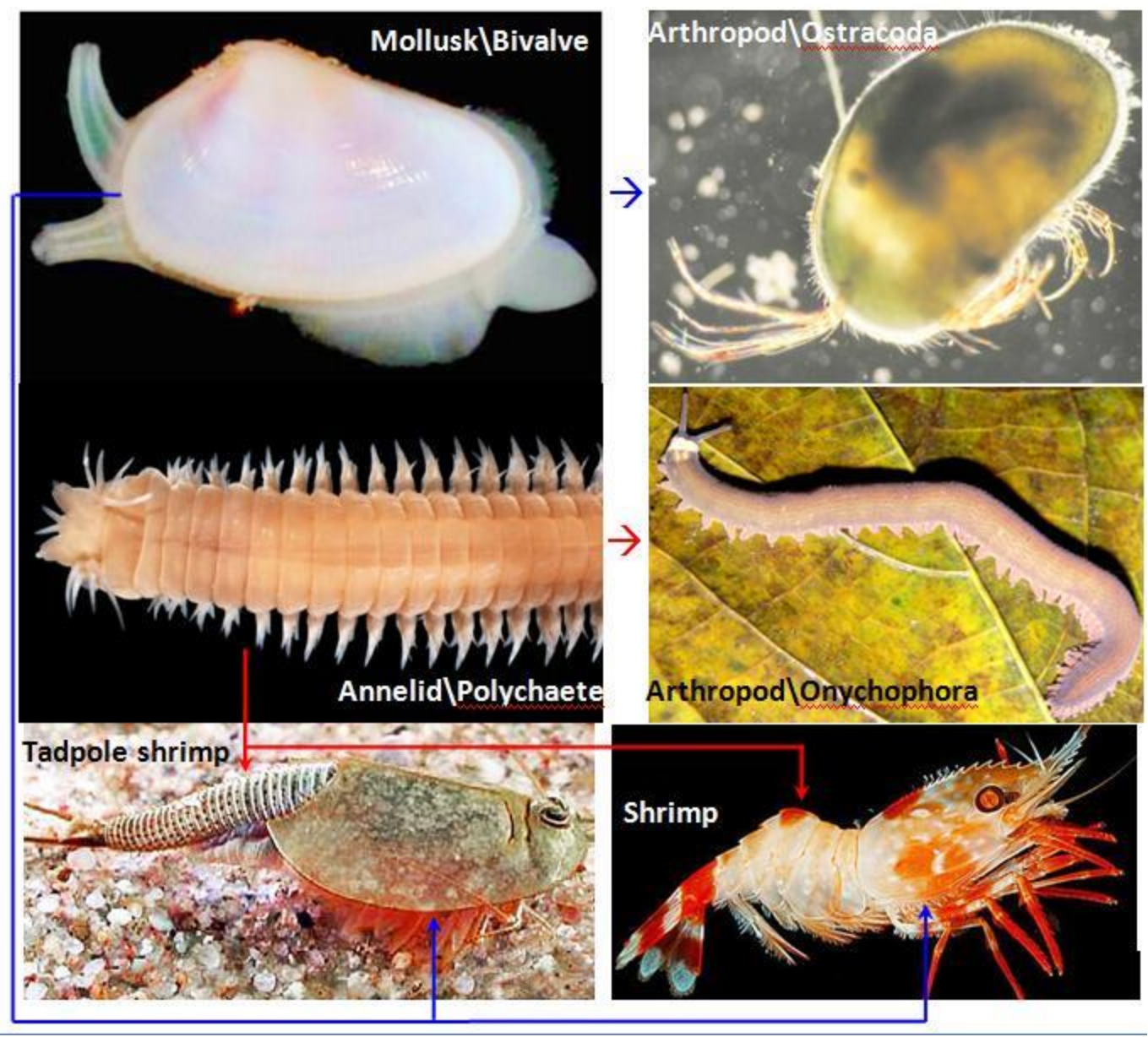

Fig. 3 The two-ancestor of crossbreeding theory is exemplified by arthropods. In arthropods, the ostracoda closely resemble the bivalve and the onychophora highly resemble the polychaete. The tadpole shrimp and the shrimp of arthropods, with the carapace of thorax came from the bivalve and the segmented abdomen came from the polychaete, is middle type, half resembling the two ancestors respectively. Mixed from many free-sources. 
The most interesting origin of ourselves came from crossbreeding between lemurs and bears, where monkeys, humans and apes comprised a spectrum. Monkeys resemble lemurs and locate at one end of the spectrum; apes resemble bears and locate at another end of the spectrum; humans are in the middle of the spectrum. The feet and teeth of humans are mainly from bears, but the hands obviously from lemurs. Both humans and apes have not a tail, which resemble the bears with a tail stub. By such a way, the evolutionary relationship of all protists and animals has been reconstructed. Some are satisfying and some require more analysis.

7. Plant evolution by crossbreeding (from further published book: Theory of Crossbreeding Evolution in Plants)

The plants in various taxon also can be arranged into spectrum as in the animals, in which two ancestors can be found. Plant species often hybridize more readily than animal species, and the resulting hybrids are fertile more often. Many plant species are the result of hybridization, combined with polyploidy, such as the above paleopolyploidy.

Here take an important example. The vascular plants are all higher plants, including ferns, conifers, and flowering plants etc. The origin of vascular tissue is especially critical. Both in the xylem and phloem, the conductive cell form vessels with perforated plates and tubes with sieve plate interrupted, for conducting water and products. These very resemble the hyphae of fungi with septa, both in structure and function. Also the branching filamentous structure is their similarity too. The wood-like section of a bracket fungus from a dead wood remind us of the highly similarity between the fungus and the wood. In crossbreeding theory, such similarity is good reason of origin. Therefore, the vascular tissue originated from the hyphae of fungi. Pteridophytes are primitive vascular plants that must inherit such important characteristic from fungi, but leaf- traits from Bryophytes.

Main evolutionary steps of plants:

1) Green Algae + Microsporidia $\rightarrow$ Bryophytes and Fungi

2) Bryophytes + Fungi $\rightarrow$ Pteridophytes

a. Hornwort + Basidiomycota $\rightarrow$ Ferns 
b. Mosses + Basidiomycota $\rightarrow$ Lycopodiophyta

c. Liverworts + Chytridiomycota(zygomycota) $\rightarrow$ Isoetopsida and Salviniales

3) Psilophyta \Trimerophytopsida +Botrychiopsis $\rightarrow$ Progymnosperms

4) Zosterophyllopsida+Marattiopsida $\rightarrow$ Pteridosperms

a. Equisetopsida +Marattiopsida $\rightarrow$ Cycadophyta, Welwitschia \& Ephedra

b. Lycopodiopsida + Ferns (Gleicheniales) $\rightarrow$ Pinophyta

5) Lyginopteridales + Cycadales $\rightarrow$ Primitive flower plants

\section{Summary}

As a sum, the all checked organisms in our global, the extinct and extant, all meet the crossbreeding theory. These are strong evidences to support this theory: the crossbreeding of two primitive ancestors produces advanced new species. The possibility of crossbreeding is always limited by the conception of sexual barrier between species, especially by different number of chromosomes between two species for exact pairing. Now, the widespread paleopolyploidy and present polyploidy tell us that it is not a problem again. It seems so easy that just need to double the genomes. The increased likelihood of large genomic reorganizations provides great advantages for forming a group of new species that is just the fan-shaped spectrum of species. Actually, crossbreeding is the rationally natural way. By this way, the origin of species becomes believable, understandable and available.

Next are some views about the crossbreeding theory.

1) According to the mutation of Darwinism, when $A$ mutated to $B, A$ must disappear; When $B$ mutated to $C, B$ must disappear...; When $Y$ mutated to $Z, Y$ must disappear. But in our global, from $A-Z$, most types are existing. From the lowest viruses and bacteria to the highest arthropods and primates, almost all types are present, although there are many extinct species.

2) Any species have two different ancestors, but not one-common ancestor. The species in the taxon have been re-arranged into fan-shaped spectrums than the phylogenic tree. The evolutionary diagram is consisted of fan-shaped spectrum in nets, but not a branched tree.

3) Species are defined by their reproductive isolation. Animal crossbreeding often occurs during mass extinction, in which solitary survivors 
copulate with others, driven by sexual pressure. Intense mating instincts drive hybrid speciation, breaking the law of reproductive isolation. Thus, a mass extinction is always accompanied by a great explosion of new species. Otherwise, the crossbreeding also can be taken place between pioneers from two groups in contacting regions for sexual attraction. Then, the sex is the cause, force and opportunity for evolution.

4) In the animal's ontogenesis, the larval form is often more like their one ancestor, while the adult form is more like another ancestor. Understanding the larval and adult forms is very important for determining the two ancestors. A few plants also have such ontogenesis.

5) A lot of species are sometimes difficult to classify. Now, according to new theory, their position can be clearly found in some spectrums.

6) The crossbreeding theory emphasizes the similarity among species, in which many genes are shared by almost all or many species, but not the differences that produced by mutation. Furthermore, the combination of genes to form new characteristics is emphasized, but not the mutation of genes to produce new characteristics. Understanding the combination and similarity, and looking for the similarity, is very important new angle for understanding of life and controlling of life.

\section{References}

1. Allendorf, F.W., Leary, R.F., Spruell, P., Wenburg, J.K. (2001). The problems with hybrids: setting conservation guidelines. Trends Ecol Evol. 16: 613-622.

2. Anderson, E. \& Hubricht, L. (1938). Hybridization in Tradescantia. III. The Evidence for Introgressive Hybridization. American Journal of Botany, 25(6): 396-402.

3. Arnold, M.L. (1997). Natural hybridization and evolution. Oxford: Oxford University Press.

4. Barton, N.H., Hewitt, G.M. (1989). Adaptation, speciation and hybrid zones. Nature, 341: 497-503.

5. Bowers, J. E., Chapman, B.A., Rong, J., Paterson, A.H. (2003). Unravelling angiosperm genome evolution by phylogenetic analysis of chromosomal duplication events. Nature, 422 (6930): 433-438.

6. Butlin, R. (1998). What do hybrid zones in general, and the Chorthippus parallelus zone in particular, tell us about speciation. Endless Forms: Species and 
Speciation (p367-389). New York: Oxford University Press.

7. Cairns, J., Overbaugh, J. , Miller, S. (1988). The origin of mutants. Nature. 335, 142 -145 .

8. Darwin, C. (1872). The Origin of species by means of natural selection, or the preservation of favoured races in the struggle for life. (the 6th edition). London.

9. Frenk, S., Pizza, G., Walker, R.V., Houseley, J. (2017). Aging yeast gain a competitive advantage on non-optimal carbon sources. Aging cell, 16(3): 602-604.

10. Garcia-Fernández, J., Holland, P.W. (1994). Archetypal organization of the amphioxus Hox gene cluster. Nature, 370: 563-566.

11. Goulet, E.B, Roda, F., Hopkins, R. (2016). Hybridization in Plants: Old Ideas, New Techniques. Plant Physiology, 173 (1): 65-78.

12. Grant, P.R. (1992). Hybridization of bird species. Science, 256: 193-197.

13. Grant, P.R., and Grant, B.R. (2014). Synergism of natural selection and introgression in the origin of a new species. Am Nat. 183(5): 671-81.

14. Harrison, R.G. (1993). Hybrid Zones and the Evolutionary Process. New York: Oxford University Press.

15. Henrique, V., Figueiró, Gang, Li., Fernanda, J., Trindade, Juliana Assis, et al. (2017). Genome-wide signatures of complex introgression and adaptive evolution in the big cats. Science Advances, 3(7): e1700299.

16. Hokamp, K., McLysaght, A., Wolfe, K.H. (2003). The $2 R$ hypothesis and the human genome sequence. Journal of Structural and Functional Genomics, 3 (1-4): 95-110.

17. Hull, R.M., Cruz, C., Jack, C.V., Houseley, J. (2017). Environmental change drives accelerated adaptation through stimulated copy number variation. PLoS biology, 15(6): e2001333.

18. Keeton, William T. (1980). Biological science. p. 800. New York: Norton.

19. Keith Adams (2013). Genomic Clues to the Ancestral Flowering Plant. Science, 342: 1456-1457.

20. Liu L.Y. (2016). New theory of species evolution. --Spectrums of species, steps of animal evolution \& speciation of crossbreeding against Darwinism. Germany, Düsseldorf: LAP Lambert Academic Publishing.

21. Lundin, L.G. (1993). Evolution of the vertebrate genome as reflected in paralogous chromosomal regions in man and the house mouse. Genomics, 16 (1): 1-19.

22. Mallet, J. (2005). Hybridization as an invasion of the genome. Trends Ecol Evol, 20: $229-237$ 
23. Mallet, J. , Beltrán, M., Neukirchen, W. , Linares, M. (2007). Natural hybridization in heliconiine butterflies: the species boundary as a continuum. BMC Evolutionary Biology, 7: 28.

24. Mayr, E. (1963). Animal Species and Evolution. Cambridge, Mass: Harvard University Press.

25. Mendel, J.G. (1865). Versuche über Pflanzenhybriden, Verhandlungen des naturforschenden Vereines in Brünn, Bd. IV für das Jahr, Abhandlungen: 3-47. For the English translation, see: Druery, C.T., Bateson, William. (1901). Experiments in plant hybridization . Journal of the Royal Horticultural Society, 26: 1-32.

26. Rong, R., Chandley, A. C., Song, J., McBeath, S., Tan, P. P., Bai, Q., Speed, R. M. (1988). A fertile mule and hinny in China. Cytogenetics and Cell Genetics. 47 (3): 134-9.

27. Seehausen, O. (2003). Hybridization and adaptive radiation. Trends Ecol Evol, 19: 198-207.

28. Smith, J.J., Keinath, M.C. (2015). The sea lamprey meiotic map improves resolution of ancient vertebrate genome duplications. Genome Res, 25 (8): 1081-90. 29. Soltis, P.S., Soltis, D.E. (2000). The role of genetic genomic attributes in the success of polyploids. PNAS. 97 (13): 7051-7057.

30. Wang, W. and Lan, H. (2000). Rapid and Parallel Chromosomal Number Reductions in Muntjac Deer Inferred from Mitochondrial DNA Phylogeny. Molecular Biology and Evolution, 17 (9): 1326-1333.

31. Witkin, E.M. (1946). Inherited differences insensitivity to radiation in scherichia Coli. Proc. Natl. 32(3): 59-68. 